\title{
Effects of inhomogeneities on apparent cosmological observables: "fake" evolving dark energy
}

\author{
Antonio Enea Romano ${ }^{1,2,3,5}$, Alexei A. Starobinsky ${ }^{4,5}$, and Misao Sasaki ${ }^{1}$ \\ ${ }^{1}$ Yukawa Institute for Theoretical Physics, \\ Kyoto University, Kyoto 606-8502, Japan \\ ${ }^{2}$ Instituto de Fisica, \\ Universidad de Antioquia, \\ A.A.1226, Medellin, Colombia \\ ${ }^{3}$ Leung Center for Cosmology and Particle Astrophysics, \\ National Taiwan University, \\ Taipei 10617, Taiwan, R.O.C. \\ ${ }^{4}$ L. D. Landau Institute for Theoretical Physics, \\ Moscow 119334, Russia \\ ${ }^{5}$ Research Centre for the Early Universe (RESCEU), \\ Graduate School of Science, \\ The University of Tokyo, \\ Tokyo 113-0033, Japan
}




\begin{abstract}
Using the exact Lemaitre-Bondi-Tolman solution with a non-vanishing cosmological constant $\Lambda$, we investigate how the presence of a local spherically-symmetric inhomogeneity can affect apparent cosmological observables, such as the deceleration parameter or the effective equation of state of dark energy (DE), derived from the luminosity distance under the assumption that the real spacetime is exactly homogeneous and isotropic. The presence of a local underdensity is found to produce apparent phantom behavior of $\mathrm{DE}$, while a locally overdense region leads to apparent quintessence behavior. We consider relatively small large scale inhomogeneities which today are not linear and could be seeded by primordial curvature perturbations compatible with CMB bounds. Our study shows how observations in an inhomogeneous $\Lambda$ CDM universe with initial conditions compatible with the inflationary beginning, if interpreted under the wrong assumption of homogeneity, can lead to the wrong conclusion about the presence of "fake" evolving dark energy instead of $\Lambda$.
\end{abstract}




\section{INTRODUCTION}

High redshift luminosity distance measurements [1 6 ] and the WMAP measurement [7, 8] of cosmic microwave background (CMB) interpreted in the context of standard FLRW cosmological models have strongly disfavored a matter dominated universe, and strongly supported a dominant dark energy component, giving rise to a positive cosmological acceleration. As an alternative to dark energy, it has been proposed [9, 10] that we may be at the center of an inhomogeneous isotropic universe described by a Lemaitre-Tolman-Bondi (LTB) solution of Einstein's field equations, where spatial averaging over one expanding and one contracting region is producing a positive averaged acceleration $a_{D}$, but is has been shown how in general this procedure can lead to formal definition of unobservable quantities [11]. Another more general approach to map luminosity distance as a function of redshift $D_{L}(z)$ to LTB models has been proposed [12], showing that an inversion method can be applied successfully to reproduce the observed $D_{L}(z)$. Interesting analysis of observational data in inhomogeneous models without dark energy is given for example in [13, 14].

The main point is that the luminosity distance is in general sensitive to the geometry of the space through which photons are propagating along null geodesics, and therefore arranging appropriately the geometry of a given cosmological model it is possible to reproduce a given $D_{L}(z)$. For FLRW models this corresponds to the determination of $\Omega_{\Lambda}$ and $\Omega_{m}$ and for LTB models it allows to determine the functions $E(r), M(r), t_{b}(r)$.

The proposal to use galaxy number counts [15] to distinguish between LTB models without cosmological constant and $\Lambda \mathrm{CDM}$ has been recently studied both analytically [16, 17] and numerically [18], showing how LTB models with a weak central singularity could in principle not be distinguished even using both the redshift spherical shell energy $m n(z)$ and $D_{L}(z)$.

In this paper we will take a different approach to the study of inhomogeneities [19 21], and instead of proposing them as an alternative to dark energy, we will consider their effects in presence of a cosmological constant, studying LTB solutions which are only locally inhomogeneous whose geometry is very closed to a $\Lambda$ CDM model, showing how even small amplitude inhomogeneities compatible with the amplitude of the curvature perturbation after inflation can lead to important effects. We in fact consider large scale inhomogeneities which today are not linear and could be seeded by primordial curvature perturbations compatible with CMB bounds. 
Since the amplitude of the inhomogeneities we consider is very small, corresponding to a few percent in terms of the density contrast, the luminosity distance $D_{L}(z)$ is not significantly affected as it can be seen in fig.(19), but the apparent cosmological observables derived from the $D_{L}(z)$ under the assumption of homogeneity are significantly affected because they are sensitive to its derivatives.

We consider different types of models of local inhomogeneities and obtain that local underdensity gives rise to apparent phantom behavior, while local overdensity leads to apparent quintessence behavior.

Our study shows how observations of a quasi- $\Lambda$ CDM universe with compensated large scale inhomogeneities compatible with inflation predictions for curvature perturbations, if interpreted under the "wrong 'assumption of homogeneity, can lead to the wrong conclusion of the presence of "fake" evolving dark energy, while only the cosmological constant is present in reality.

\section{DERIVING THE EXACT LTB SOLUTION WITH A COSMOLOGICAL CON- STANT}

The LTB solution can be written as $[22-24]$ as

$$
d s^{2}=-d t^{2}+\frac{\left(R,{ }_{r}\right)^{2} d r^{2}}{1+2 E(r)}+R^{2} d \Omega^{2},
$$

where $R$ is a function of the time coordinate $t$ and the radial coordinate $r, E(r)$ is an arbitrary function of $r$, and $R_{, r}=\partial_{r} R(t, r)$.

The Einstein equations with dust and a cosmological constant give

$$
\begin{aligned}
& \left(\frac{\dot{R}}{R}\right)^{2}=\frac{2 E(r)}{R^{2}}+\frac{2 M(r)}{R^{3}}+\frac{\Lambda}{3}, \\
& \rho(t, r)=\frac{2 M,_{r}}{R^{2} R_{, r}},
\end{aligned}
$$

with $M(r)$ being an arbitrary function of $r, \dot{R}=\partial_{t} R(t, r)$ and $c=8 \pi G=1$ is assumed throughout the paper. Since Eq. (2) contains partial derivatives respect to time only, its general solution can be obtained from the FLRW equivalent solution by making every constant in the latter one an arbitrary function of $r$. Previous studies of LTB solutions in presence of a cosmological constant include [25 27]. The general analytical solution for a FLRW model with dust and cosmological constant was obtained by Edwards [28] in terms of 
elliptic functions. By an appropriate choice of variables and coordinates, we may extend it to the LTB case thanks to the spherical symmetry of both LTB and FLRW models, and to the fact that dust follows geodesics without being affected by adjacent regions. The Friedman equation for the scale factor $a_{F}(t)$ of a pressureless FLRW universe with a cosmological constant has the form:

$$
\left(\frac{\dot{a_{F}}}{a_{F}}\right)^{2}=-\frac{k}{a_{F}^{2}}+\frac{\rho_{0}}{3 a_{F}^{3}}+\frac{\Lambda}{3}
$$

It is convenient to introduce the conformal time $\eta$ such that $d \eta=d t / a_{F}$, in terms of which the solution satisfying the initial Big-Bang condition $a_{F}(0)=0$ can then be expressed as

$$
a_{F}(\eta)=\frac{\rho_{0}}{3 \phi\left(\frac{\eta}{2} ; g_{2}, g_{3}\right)+k} ; \quad g_{2}=\frac{4}{3} k^{2}, \quad g_{3}=\frac{4}{27}\left(2 k^{3}-\Lambda \rho_{0}^{2}\right)
$$

where $\phi\left(x ; g_{2}, g_{3}\right)$ is the Weierstrass elliptic function satisfying the differential equation,

$$
\left(\frac{d \phi}{d x}\right)^{2}=4 \phi^{3}-g_{2} \phi-g_{3}
$$

We note that in [28], the curvature parameter $k$ is normalized to $k= \pm 1$, hence $k^{2}=1$. However, for our purpose below we present the solution without normalizing $k^{2}$ to unity. We can now use this solution to construct a general solution of the partial differential equation (2). First, we introduce a new coordinate $\eta=\eta(t, r)$ and a variable $a$ by

$$
\left(\frac{\partial \eta}{\partial t}\right)_{r}=\frac{r}{R} \equiv \frac{1}{a}
$$

and introduce new functions by

$$
\rho_{0}(r) \equiv \frac{6 M(r)}{r^{3}}, \quad k(r) \equiv-\frac{2 E(r)}{r^{2}}
$$

Then Eq. (2) becomes

$$
\left(\frac{\partial a}{\partial \eta}\right)^{2}=-k(r) a^{2}+\frac{\rho_{0}(r)}{3} a+\frac{\Lambda}{3} a^{4}
$$

where $a$ is now regarded as a function of $\eta$ and $r ; a=a(\eta, r)$. It should be noted that the coordinate $\eta$, which is a generalization of the conformal time in a homogeneous FLRW universe, has been only implicitly defined by Eq. (7). The actual relation between $t$ and $\eta$ can be obtained by integration $t=\int a d \eta$ once $a(\eta, r)$ is known. Inspired by the construction of the solution for the FLRW case, we finally get

$$
a(\eta, r)=\frac{\rho_{0}(r)}{3 \phi\left(\frac{\eta}{2} ; g_{2}(r), g_{3}(r)\right)+k(r)} .
$$




\section{GEODESIC EQUATIONS}

We adopt the same method developed in [29] to solve the null geodesic equation written in terms of the coordinates $(\eta, r)$. Instead of integrating differential equations numerically, we perform a local expansion of the solution around $z=0$ corresponding to the point $\left(t_{0}, 0\right)$, or equivalently $\left(\eta_{0}, 0\right)$, where $t_{0}=t\left(\eta_{0}, 0\right)$. The change of variables from $(t, r)$ to $(\eta, r)$ permits us to have r.h.s. of all equations in a fully analytical form, in contrast to previous considerations of this problem which require a numerical calculation of $R(t, r)$ from the Einstein equation (2). Thus, this formulation is particularly suitable for derivation of analytical results.

The luminosity distance for a central observer in the LTB space-time as a function of the redshift $z$ is expressed as

$$
D_{L}(z)=(1+z)^{2} R(t(z), r(z))=(1+z)^{2} r(z) a(\eta(z), r(z))
$$

where $(t(z), r(z))$ or $((\eta(z), r(z))$ is the solution of the radial geodesic equation as a function of $z$. The past-directed radial null geodesics is given by

In terms of $z$, Eq. (12) takes the form [30]:

$$
\frac{d t}{d r}=-\frac{R_{, r}(t, r)}{\sqrt{1+2 E(r)}} .
$$

$$
\begin{aligned}
\frac{d r}{d z} & =\frac{\sqrt{1+2 E(r(z))}}{(1+z) \dot{R}_{, r}[r(z), t(z)]}, \\
\frac{d t}{d z} & =-\frac{R_{, r}[r(z), t(z)]}{(1+z) \dot{R}_{, r}[r(z), t(z)]} .
\end{aligned}
$$

The inconvenience of using the $(t, r)$ coordinates is that there is no exact analytical solution for $R(t, r)$. So the r.h.s. of Eqs. (13) cannot be evaluated analytically, but we are required to find a numerical solution for $R$ first [31], and then to integrate numerically the differential equations, which is quite an inconvenient and cumbersome procedure. Alternatively one may derive a local expansion of $R(t, r)$ around $\left(t_{0}, 0\right)$, corresponding to the central observer, and use it Eqs. (13). But one would need to expand it to a higher order in $z$ in order to maintain the accuracy at high redshifts.

For this reason, it is useful for many numerical and analytical applications to write the geodesic equations in terms of the coordinates $(\eta, r)$. It follows from the definition (7) that

$$
t(\eta, r)=t_{b}(r)+\int_{0}^{\eta} a\left(\eta^{\prime}, r\right) d \eta^{\prime}
$$


hence,

$$
d t=a(\eta, r) d \eta+\left(\int_{0}^{\eta} \frac{\partial a\left(\eta^{\prime}, r\right)}{\partial r} d \eta^{\prime}+t_{b}^{\prime}(r)\right) d r
$$

Partial derivatives are transformed using the relations:

$$
\left(\frac{\partial}{\partial t}\right)_{r}=a^{-1}\left(\frac{\partial}{\partial \eta}\right)_{r}, \quad\left(\frac{\partial}{\partial r}\right)_{t}=\left(\frac{\partial}{\partial r}\right)_{\eta}-a^{-1}\left(\frac{\partial t}{\partial r}\right)_{\eta}\left(\frac{\partial}{\partial \eta}\right)_{r} .
$$

Then Eqs. (13) take the form:

$$
\begin{aligned}
& \frac{d \eta}{d z}=-\frac{\partial_{r} t(\eta, r)+F(\eta, r)}{(1+z) \partial_{\eta} F(\eta, r)} \equiv p(\eta, r), \\
& \frac{d r}{d z}=\frac{a(\eta, r)}{(1+z) \partial_{\eta} F(\eta, r)} \equiv q(\eta, r),
\end{aligned}
$$

where

$$
F(\eta, r) \equiv \frac{R_{, r}}{\sqrt{1+2 E(r)}}=\frac{1}{\sqrt{1-k(r) r^{2}}}\left[\partial_{r}(a(\eta, r) r)-a^{-1} \partial_{\eta}(a(\eta, r) r) \partial_{r} t(\eta, r)\right] .
$$

It is important that the functions $p, q, F$ have explicit analytical forms.

\section{APPARENT COSMOLOGICAL OBSERVABLES AND "FAKE" DARK EN- ERGY}

In this section we will briefly introduce the concept of apparent observables, which are deduced from observations assuming a flat $\Lambda$ CDM model. We start from observing that in a flat FLRW model [32] there are simple relations between the Hubble parameter $H(z)$, the luminosity distance $D_{L}(z)$, the cosmic deceleration $q(z)$ and the effective equation of state of dark energy $w_{D E}(z)$ :

$$
\begin{array}{r}
H^{F L R W}(z)=\left[\frac{d}{d z}\left(\frac{D_{L}^{F L R W}(z)}{1+z}\right)\right]^{-1} . \\
Q^{F L R W}(z)=\frac{d}{d z}\left(\frac{D_{L}^{F L R W}(z)}{1+z}\right)=\left(H^{F L R W}(z)\right)^{-1}, \\
q^{F L R W}(z)=-1-\frac{d \ln \left(Q^{F L R W}(z)\right)}{d \ln (1+z)}=q^{F L R W}\left(D_{L}(z)\right), \\
w_{D E}^{F L R W}(z)=\frac{(2(1+z) / 3) d \ln H^{F L R W} / d z-1}{1-\left(H_{0} / H^{F L R W}\right)^{2}, \Omega_{0 m}(1+z)^{3}} .
\end{array}
$$

We will use the above relations to define apparent observables in terms of the observed luminosity distance according to

$$
H^{a p p}(z)=\left[\frac{d}{d z}\left(\frac{D_{L}^{o b s}(z)}{1+z}\right)\right]^{-1}
$$




$$
\begin{array}{r}
Q^{a p p}(z)=\frac{d}{d z}\left(\frac{D_{L}^{o b s}(z)}{1+z}\right)=\left(H^{a p p}(z)\right)^{-1}, \\
q^{a p p}(z)=-1-\frac{d \ln \left(Q^{a p p}(z)\right)}{d \ln (1+z)}=q^{a p p}\left(D_{L}^{o b s}(z)\right), \\
w_{D E}^{a p p}(z)=\frac{(2(1+z) / 3) d \ln H^{a p p} / d z-1}{1-\left(H_{0} / H^{a p p}\right)^{2} \Omega_{0 m}(1+z)^{3}} .
\end{array}
$$

where $q^{a p p}(z)$ is the apparent cosmic deceleration parameter, $w_{D E}^{a p p}(z)$ the apparent equation of state of dark energy and $Q^{a p p}(z)$ is an auxiliary function introduced of mathematical convenience. Apparent observables are deduced from the observed luminosity distance assuming the same functional relations which apply to the case of a homogeneous and isotropic universe described by a FLRW metric. We will apply these definitions of apparent observables assuming the observed luminosity distance corresponds to the case of a central observer in a $\Lambda$ LTB space. If the Universe is really inhomogeneous the apparent observables above will include the errors due to ignoring the inhomogeneity, which could for example be mistaken as dark energy with a redshift dependent equation of state, that is, we may be fooled by 'fake' dark energy.

In this paper in addition to the above, we will also consider $\operatorname{Om}(z)$ [33, 34], a diagnostic which can be used to distinguish $\Lambda \mathrm{CDM}$ from other DE models without directly involving the cosmic equation of state,

$$
O m^{a p p}(x) \equiv \frac{h^{2}(x)-1}{x^{3}-1}, \quad x=1+z, h(x)=H^{a p p}(x) / H_{0} .
$$

\section{EFFECTS OF LOCAL INHOMOGENEITIES ON APPARENT OBSERVABLES}

In order to make a connection between the LTB model and a universe with primordial curvature perturbations from inflation, we introduce the following metric which describes a spherically symmetric space-time after inflation on scales much exceeding the Hubble scale:

$$
d s^{2}=-d t^{2}+a_{F}^{2}(t) e^{2 \zeta(r)}\left(d r^{2}+r^{2} d \Omega^{2}\right) .
$$

According to the inflationary scenario, $\zeta(r)$ is just a local, space-dependent number of $e$ folds $N$ produced during inflation (up to a constant which may be absorbed into $a_{F}$ ). This relation which constitutes the basis of the so-called $\delta N$ formalism was first obtained in [35] in case of a single field inflation, and then generalized to multiple field inflation in [36, 37]; see also [39] for further consideration. 
This metric in general can describe a stage dominated by any form of the matter with any equation of state. But it is valid only for inhomogeneities whose characteristic scale $L$ is much greater than the Hubble horizon scale, $H L \gg 1$. In other words, it is the metric valid at leading order in the spatial gradient expansion [39]. Since we have $L=a_{F} \ell$ where $\ell$ is the comoving scale, while $H$ scales as $a_{F}^{-2}$ during the radiation-dominated stage or $a_{F}^{-3 / 2}$ during the matter-dominated stage, $H L=H a_{F} \ell=\dot{a}_{F} \ell$ is a decreasing function of time. So for a given comoving scale of inhomogeneities $\ell$, the metric (29) is valid only at an early stage of the universe when $\dot{a}_{F} \ell \gg 1$.

On the other hand, the LTB metric is valid on any scales but only after the universe has become matter-dominated. Fortunately, for sufficiently large scale inhomogeneities, say those whose scale is $1 / 10$ of the current Hubble radius, which is the case for specific examples studied below, there is a sufficiently wide overlap of time during which both metrics are valid, after the recombination of hydrogens at the redshift $z \sim 10^{3}$ until the redshift $\sim 100$.

The important point is that this identification allows us to make a direct connection between the primordial curvature perturbations encoded in $\zeta(r)$ and the function $k(r)$ appearing in the LTB solution. In this way we can justify the size of the present day local large scale inhomogeneity we consider by relating it to early time curvature perturbations, making our model more realistic. Since the metric (29) is valid on super-Hubble scales, at the time of the matching the perturbations seeding our present day inhomogeneity were super-horizon, but today has become sub-horizon.

Matching the metric (29) with the LTB metric at the stage when both are valid as descibed above, we obtain the following relations:

$$
\begin{aligned}
R & =a_{F}(t) e^{\zeta(r)} r, \\
1+2 E(r) & =\left[1+r \zeta^{\prime}(r)\right]^{2} .
\end{aligned}
$$

We note that the first equality is only approximate. It ignores corrections of the order $(H L)^{-2}$. But the second equality is exact in the sense that the amplitude of $\zeta$ does not have to be small, though it is small in reality. At the linear approximation, this reduces to

$$
k(r)=-2 \frac{\zeta^{\prime}(r)}{r} .
$$

In particular, $k(0)=-2 \zeta^{\prime \prime}(0)$. Note that the LTB metric (1) or the relation (31) is invariant under the change of the radial coordinate $r \rightarrow \bar{r}=g(r)$, where $g(r)$ is an arbitrary function of $r$ as long as it is monotonic in $r$. We will choose the coordinates in which $\rho_{0}=$ constant. 
This approach has the goal to establish some quantitative bounds on the size of a spherically symmetric inhomogeneity which may surround us and is compatible with the inflationary predictions. Using the above relations we can in fact directly relate the function $k(r)$, which in the coordinates we chose completely determine an LTB model, with the primordial curvature perturbations produced by inflation. This makes our models more realistic since we will study inhomogeneities which are compatible with inflation predictions.

Motivated by observations we consider the curvature perturbation $\zeta(r)$ of the amplitude $\sim 5 \times 10^{-5}$. Specifically we study the four different types of inhomogeneities,

$$
\begin{array}{ll}
\text { Type } \mathrm{I}^{-}: k(r)=\frac{A}{r_{0}^{2}}\left[l(r)-l^{\prime}(0) r e^{-r / r_{0}}\right] ; & H_{0} r_{0}=0.1, A=10^{-4}, \Delta=0.02, \\
{\text { Type } \mathrm{I}^{+}:}(r)=\frac{A}{r_{0}^{2}}\left[l(r)-l^{\prime}(0) r e^{-r / r_{0}}\right] ; & H_{0} r_{0}=0.1, A=-10^{-4}, \Delta=0.02, \\
\text { Type } \mathrm{II}^{-}: \zeta(r)=A\left[l(r)-l^{\prime}(0) r e^{-r / r_{0}}\right] ; & H_{0} r_{0}=0.2, A=5 \times 10^{-5}, \Delta=0.05, \\
\text { Type } \mathrm{II}^{+}: \zeta(r)=A\left[l(r)-l^{\prime}(0) r e^{-r / r_{0}}\right] ; & H_{0} r_{0}=0.2, A=-5 \times 10^{-5}, \Delta=0.05,
\end{array}
$$

where the function $l(r)$ is defined as

$$
l(r)=\left[\tanh \left(\frac{H_{0}\left(r-r_{0}\right)}{\Delta}\right)-1\right] .
$$

In all cases, the cosmological constant is assumed to be the same as the one implied by the best fit $\Lambda$ CDM model corresponding to $\Omega_{\Lambda}=0.7$, and $H_{0}=H^{a p p}(z=0)$ is adjusted to the observed Hubble constant.

We have chosen these four different inhomogeneity profiles because they correspond to compensated inhomogeneities, in the sense that they asymptotically approach a flat, homogeneous $\Lambda$ CDM model and correspond, respectively, to

- Type $\mathrm{I}^{+}$: central overdense region.

- Type $\mathrm{I}^{-}$: central underdense region.

- Type $\mathrm{II}^{+}$: intermediate overdense region.

- Type $\mathrm{II}^{-}$: intermediate underdense region.

An important feature of type I models is that they are by construction regular at the center, since the linear term in the series expansion at the center is removed, avoiding the cusp singularity which would otherwise arise. Type II models are also regular at the center, i.e. 
$k(r)$ and $k^{\prime}(r)$ do not diverge, though it is not immediately apparent. It can be seen by expanding $\zeta(r)$ in qqs.(36) (36) around $r=0$.

As seen from Figs. (1-9) for type $\mathrm{I}^{ \pm}$models, the presence of a local underdensity gives rise to apparent phantom behavior, while that of a local overdense region to apparent quintessence behavior. For a shell-like underdensity or overdensity region, Figs. (10 18) for type $\mathrm{II}^{ \pm}$also indicate that an underdensity shell mimics phantom behavior, while an overdensity shell mimics quintessence behavior.

Since all the models considered here describe compensated inhomogeneities, they show both phantom and quiescence behaviors, but we can see that the sign of the variation of $w_{D E}^{a p p}$ with respect to $w_{\text {true }}=-1$ is roughly the same as the sign of the density contrast.

Our results give a semi-realistic example of inhomogeneities compatible with observations which, if interpreted in the framework of a flat and inhomogeneous spacetime, can lead to the wrong conclusion that there exists dark energy with an evolving equation of state, while in reality there is only a cosmological constant.

The relation between density profiles and the free parameters of the LTB metric is quite difficult and elaborate, as shown for example in [38, 40]. One useful way to analyze the relation between the profile of $k(r)$ with that of $\rho(r)$ is to consider the linear theory limit. On sufficiently large scales where the amplitude of $\zeta$ is small, $\zeta \ll 1$, and its spatial variation is sufficiently smooth, $r\left|\zeta^{\prime}(r)\right| \ll 1, \zeta$ is equal to the conserved comoving curvature perturbation in linear theory, which it is essentially equal to the minus of the Newton potential $\Psi$ in the Newton gauge. At the matter-dominated stage, $\Psi=-(3 / 5) \zeta$. So we can estimate the resulting density profile for a given profile of $\zeta$ from the Poisson equation,

$$
\delta \rho \propto \Delta \Psi \propto \frac{3}{10} r^{-2} \frac{d\left(r^{3} k(r)\right)}{d r}
$$

where we have used the fact that in the limit $r\left|\zeta^{\prime}(r)\right| \ll 1$ eq.(28) holds. Although this is valid only at linear order in the strict sense, it gives the correct qualitative behavior of the density profile. This indeed shows that the sign of $k(r)$ is an important factor but not always the dominant factor in the determination of the density profile.

\section{ANALYTICAL DERIVATION OF $q_{0}^{a p p}$ AND $w_{0}^{a p p}$}

The calculation of the central value of the apparent cosmological observables requires to find an analytical expression for the right hand side of the geodesics equations. For this 
purpose we expand the relevant functions as

$$
\begin{aligned}
t(\eta, r) & =A_{0}(\eta)+A_{1}(\eta) r+\frac{1}{2} A_{2}(\eta) r^{2}+\cdots \\
\eta(z) & =\eta_{0}+\eta_{1} z+\eta_{2} z^{2}+\cdots \\
r(z) & =r_{1} z+r_{2} z^{2}+\cdots
\end{aligned}
$$

to get

$$
\begin{aligned}
q_{0}^{a p p} & =-\frac{2\left(r_{1} a_{, r}+\eta_{1} a_{, \eta}\right)}{a}-\frac{2 r_{2}}{r_{1}}-3, \\
w_{0}^{a p p} & =-\frac{4 r_{1}\left(r_{1} a_{, r}+\eta_{1} a_{, \eta}\right)+\left(7 r_{1}+4 r_{2}\right) a}{3 r_{1} a\left(1-H_{0}^{2} \Omega_{M}\left(r_{1} a\right)^{2}\right)} .
\end{aligned}
$$

The solution of the geodesics equations leads to

$$
\begin{aligned}
& r_{1}=\frac{a^{0}}{a_{, \eta}^{0}} \\
& r_{2}=\frac{a\left(a_{, \eta} A_{1}^{\prime}\left(\eta_{0}\right)+2 A_{1}\left(\eta_{0}\right) a_{, \eta \eta}-2 a_{, \eta}^{2}+a_{, r} a_{, \eta}\right)-2 A_{1}\left(\eta_{0}\right) a_{, \eta}^{2}+\left(a_{, \eta \eta}-2 a_{, \eta r}\right) a^{2}}{2 a_{, \eta}^{3}} \\
& \eta_{1}=-\frac{a^{0}+A_{1}\left(\eta_{0}\right)}{a_{, \eta}^{0}}
\end{aligned}
$$

We can then also expand the energy density around the center,

$$
\rho(\eta, r)=\frac{\rho_{0}}{a(\eta, 0)^{3}}+\frac{r \rho_{0}\left[A_{1}(\eta) a_{, \eta}(\eta, 0)-4 a(\eta, 0) a_{, r}(\eta, 0)\right]}{a(\eta, 0)^{5}}+O\left(r^{2}\right) .
$$

In order to avoid a central singularity the term linear in $r$ should vanish, and from this condition we obtain

$$
A_{1}(\eta)=\frac{4 a(\eta, 0) a_{, r}(\eta, 0)}{a_{, \eta}(\eta, 0)}
$$

Using this equation we finally get

$$
\begin{aligned}
q_{0}^{a p p} & =\frac{a_{, r}\left(9 a_{, \eta}^{2}-4 a a_{, \eta \eta}\right)+a_{, \eta}\left(a_{, \eta}^{2}-a\left(2 a_{, \eta r}+a_{, \eta \eta}\right)\right)}{a_{\eta}^{3}}, \\
w_{0}^{a p p} & =\frac{a_{, r}\left(8 a a_{, \eta \eta}-18 a_{, \eta}^{2}\right)+a_{, \eta}\left(2 a\left(2 a_{, \eta r}+a_{, \eta \eta}\right)-a_{, \eta}^{2}\right)}{3 a_{, \eta}\left(H_{0}^{2} \Omega_{M} a^{4}-a_{, \eta}^{2}\right)},
\end{aligned}
$$

where the right-hand sides are evaluated at $(\eta, r)=\left(\eta_{0}, 0\right)$. It may be worth mentioning that the formulas derived so far are general in the sense they do not depend on the explicit form of the solution. 
We can also define

$$
q_{0}=-\frac{\ddot{a}\left(t_{0}, 0\right) \dot{a}\left(t_{0}, 0\right)}{\dot{a}\left(t_{0}, 0\right)^{2}},
$$

where the derivative respect to $t$ is denoted with a dot, and is calculated using the analytical solution $a(\eta, r)$ and the derivative respect to $\eta$,

$$
\dot{a}=\partial_{t} a=\frac{\partial_{\eta} a}{a} .
$$

It is interesting to observe that because of the regularity condition we have imposed at the center, we have

$$
q_{0}^{a p p}=q_{0}
$$

which can be verified using the analytical solution both for the case of vanishing and non vanishing cosmological constant.

Using again the condition $k_{1}=0$ we can now substitute the analytical solution to get the final results expressed directly in terms of observables:

$$
\begin{aligned}
q_{0}^{a p p} & =\frac{3}{2} \Omega_{M}-1+2 \tilde{\zeta^{\prime \prime}}(0), \\
w_{0}^{a p p} & =-1+\frac{4}{3\left(1-\Omega_{M}\right)} \tilde{\zeta^{\prime \prime}}(0),
\end{aligned}
$$

where we have used

$$
\begin{aligned}
a_{0} & =\frac{L^{2} \rho_{0}}{\zeta^{\prime \prime}(0) L^{2}+3 \phi_{0}}, \\
H_{0} & =-\frac{3 \phi_{0}^{\prime}}{2 L^{3} \rho_{0}}, \\
\phi_{0} & =\phi\left(\frac{\eta_{0}}{2 L} ; \frac{16}{3} \zeta^{\prime \prime}(0)^{2} L^{4},-\frac{4}{27}\left(16 \zeta^{\prime \prime}(0)^{3}+\Lambda \rho_{0}^{2}\right) L^{6}\right), \\
\phi_{0}^{\prime} & =\left.\partial_{x} \phi\left(x ; \frac{16}{3} \zeta^{\prime \prime}(0)^{2} L^{4},-\frac{4}{27}\left(16 \zeta^{\prime \prime}(0)^{3}+\Lambda \rho_{0}^{2}\right) L^{6}\right)\right|_{x=\frac{\eta_{0}}{2 L}}, \\
\Lambda & =3\left(1-\Omega_{M}\right) H_{0}^{2}, \\
\zeta^{\prime \prime}(0) & =\frac{1}{\left(a_{0} H_{0}\right)^{2}} \zeta^{\prime \prime}(0), \\
\rho_{0} & =3 a_{0}^{3} \Omega_{M} H_{0}^{2} .
\end{aligned}
$$

As expected the above formulae reduce to the $\Lambda C D M$ case in the central flat limit,

$$
\begin{aligned}
k_{0}=-2 \zeta^{\prime \prime}(0) & =0, \\
q_{0}^{a p p}=q_{0}^{\Lambda C D M} & =\frac{3}{2} \Omega_{M}-1, \\
w_{0}^{a p p}=w_{0}^{\Lambda C D M} & =-1 .
\end{aligned}
$$




\begin{tabular}{|l|l|l|}
\hline Type & $q_{0}^{a p p}$ & $w_{0}^{a p p}$ \\
\hline$\Lambda C D M$ & -0.58 & -1 \\
\hline I & -0.56001 & 0.981482 \\
\hline II & -0.599999 & -1.01852 \\
\hline III & -0.579866 & -0.999876 \\
\hline IV & -0.580134 & -1.00012 \\
\hline
\end{tabular}

TABLE I: The apparent value of $q_{0}$ and $w_{0}$ is given using the analytical formula in eq.( 55555). In all the five models $\Omega_{\Lambda}=0.72$. The $\Lambda C D M$ case is reported for reference for reference to the standard cosmological model.

As a confirmation that large scale inhomogeneities look like fake dark energy we can also verify that the relation between $q_{0}^{a p p}$ and $w_{0}^{a p p}$ is the same as in the case of an FLRW model with dark energy:

$$
\begin{aligned}
q_{0}^{F L R W} & =\frac{3}{2} \Omega_{m}-1+\frac{3}{2}\left(1+w_{0}^{D E}\right)\left(1-\Omega_{M}\right), \\
q_{0}^{\text {app }} & =\frac{3}{2} \Omega_{m}-1+\frac{3}{2}\left(1+w_{0}^{a p p}\right)\left(1-\Omega_{M}\right) .
\end{aligned}
$$

It should be noted that the above relations are general since they do not depend on the particular type of inhomogeneity profile, follow directly from the definition of $w^{a p p}$ and $q^{a p p}$ and have been derived to show that a large scale inhomogeneity self-consistently mimick evolving dark energy.

\section{CONCLUSIONS}

We have investigated how the presence of a local inhomogeneity could affect the apparent equation of state of dark energy under the "wrong" assumption of a homogeneous FLRW background, which is commonly used in interpreting astrophysical observations in $\Lambda \mathrm{CDM}$ models. Our calculation shows how phantom and quintessence behaviors can be produced for compensated underdense or overdense regions. The presence of a local underdensity gives rise to apparent phantom behavior, while that of a local overdense region to apparent quintessence behavior.

Our results give a semi-realistic example of inhomogeneities with the amplitude compatible with inflationary predictions which, if interpreted in the framework of a flat and 
inhomogeneous spacetime, can lead to the wrong conclusion of the presence of dark energy with an evolving equation of state. In general, a local inhomogeneity can lead to a confusion between local gravitational redshift and cosmological redshift due to the expansion of the Universe.

Recent analysis of observational data [41] could support the existence of a local underdense region, but which may not be of compensated type as the one we considered here. We will investigate in a future work what could be the constraints on the size and density contrast of such a void based on observational data.

\section{Acknowledgments}

AER is supported by MEXT Grant-in-Aid for the global COE program at Kyoto University, "The Next Generation of Physics, Spun from Universality and Emergence", by the UDEA research project IN615CE and the dedicacion exclusiva program. MS is supported in part by JSPS Grant-in-Aid for Scientific Research (A) No. 21244033, and by JSPS Grant-inAid for Creative Scientific Research No. 19GS0219. AS acknowledges RESCEU hospitality as a visiting professor. He was also partially supported by the grant RFBF 11-02-00643 and by the Scientific Programme "Astronomy" of the Russian Academy of Sciences.

\section{Appendix A: Calculating the density contrast}

In the text, we have carried out all our calculations in the coordinates $(\eta, r)$ since this allows to take full advantage of the existence of an analytical solution. But if we are interested in the radial profile of a quantity on a fixed time-slice $t=$ constant, we need to go back to the coordinates $(t, r)$. Below we carry this out for the density contrast, $\delta=(\rho(t, r)-\rho(t, \infty)) / \rho(t, \infty)$, where our LTB model is assumed to approach a flat FLRW universe as $r \rightarrow \infty$.

We need to introduce the inverse of the function defined in eq. (14), i.e., we need to express $\eta$ as a function of $(t, r), \eta=v(t, r)$, from

$$
t=u(\eta, r)=t_{b}(r)+\int_{0}^{\eta} a\left(\eta^{\prime}, r\right) d \eta^{\prime}
$$

such that

$$
u(v(t, r), r)=t .
$$


The value of $v(t, r)$ can be evaluated numerically by solving for $x$ the equation

$$
u(x, r)=t
$$

The function $\eta=v\left(t_{0}, r\right)$ thus obtained is plotted for the different models in Figs. 4 and 13 , As it can be seen, $\eta=v\left(t_{0}, r\right)$ varies substantially in the region of inhomogeneity, while it levels off to a constant far from the inhomogeneity.

The energy density in the coordinates $(t, r)$ is given by

$$
\rho(t, r)=\frac{2 \partial_{r} M}{R^{2}(t, r) \partial_{r} R(t, r)}
$$

But since the analytical solution is given in terms of $\eta$ we have another expression,

$$
\rho(\eta, r)=\frac{2 \partial_{r} M}{a(\eta, r)^{2} r^{2}\left[\partial_{r}(a(\eta, r) r)-a^{-1} \partial_{\eta}(a(\eta, r) r) \partial_{r} t\right]} .
$$

Then the density contrast on the hypersurface $t=t_{0}$ is given by

$$
\delta\left(t_{0}, r\right)=\frac{\rho\left(\eta\left(t_{0}, r\right), r\right)-\rho\left(\eta\left(t_{0}, \infty\right), \infty\right)}{\left.\rho\left(\eta\left(t_{0}, \infty\right)\right), \infty\right)}
$$

where

$$
t_{0}=t\left(\eta_{0}, 0\right)
$$

The density contrast is plotted in Fig. 5 for type $\mathrm{I}^{ \pm}$inhomogeneities and Fig. 14 for type $\mathrm{II}^{ \pm}$inhomogeneities.

[1] A. G. Riess et al. [Supernova Search Team Collaboration], Astron. J. 116, 1009 (1998) arXiv:astro-ph/9805201.

[2] J. L. Tonry et al. [Supernova Search Team Collaboration], Astrophys. J. 594, 1 (2003) arXiv:astro-ph/0305008.

[3] R. A. Knop et al. [The Supernova Cosmology Project Collaboration], Astrophys. J. 598, 102 (2003) arXiv:astro-ph/0309368.

[4] B. J. Barris et al., Astrophys. J. 602, 571 (2004) arXiv:astro-ph/0310843.

[5] A. G. Riess et al. [Supernova Search Team Collaboration], Astrophys. J. 607, 665 (2004) arXiv:astro-ph/0402512. 
[6] S. Perlmutter et al. [Supernova Cosmology Project Collaboration], "Measurements of Omega and Lambda from 42 High-Redshift Supernovae," Astrophys. J. 517, 565 (1999) arXiv:astro-ph/9812133.

[7] C. L. Bennett et al., Astrophys. J. Suppl. 148, 1 (2003) arXiv:astro-ph/0302207];

[8] D. N. Spergel et al. [WMAP Collaboration], Astrophys. J. Suppl. 170, 377 (2007) astro-ph/0603449.

[9] Y. Nambu and M. Tanimoto, arXiv:gr-qc/0507057.

[10] T. Kai, H. Kozaki, K. i. nakao, Y. Nambu and C. M. Yoo, Prog. Theor. Phys. 117, 229 (2007) arXiv:gr-qc/0605120.

[11] A. E. Romano, Phys. Rev. D 75, 043509 (2007) arXiv:astro-ph/0612002.

[12] D. J. H. Chung and A. E. Romano, Phys. Rev. D 74, 103507 (2006) arXiv:astro-ph/0608403.

[13] S. Alexander, T. Biswas, A. Notari and D. Vaid, JCAP 0909, 025 (2009) arXiv:0712.0370 [astro-ph]].

[14] H. Alnes, M. Amarzguioui and O. Gron, Phys. Rev. D 73, 083519 (2006) arXiv:astro-ph/0512006.

[15] A. E. Romano, Phys. Rev. D 76, 103525 (2007) arXiv:astro-ph/0702229.

[16] A. E. Romano, JCAP 1001, 004 (2010) arXiv:0911.2927 [astro-ph.CO]].

[17] A. E. Romano, JCAP 1005, 020 (2010) [arXiv:0912.2866 [astro-ph.CO]].

[18] M. -N. Celerier, K. Bolejko and A. Krasinski, Astron. Astrophys. 518, A21 (2010) arXiv:0906.0905 [astro-ph.CO]].

[19] A. E. Romano and P. Chen, JCAP 1110, 016 (2011) [arXiv:1104.0730 [astro-ph.CO]].

[20] A. E. Romano and P. Chen, arXiv:1207.5572 [astro-ph.CO].

[21] A. E. Romano and P. chen, Int. J. Mod. Phys. D 20, 2823 (2011) [arXiv:1208.3911 [gr-qc]].

[22] G. Lemaitre, Annales Soc. Sci. Brux. Ser. I Sci. Math. Astron. Phys. A 53, 51 (1933).

[23] R. C. Tolman, Proc. Nat. Acad. Sci. 20, 169 (1934).

[24] H. Bondi, Mon. Not. Roy. Astron. Soc. 107, 410 (1947).

[25] G C Omer, Proc Natl Acad Sci 53, 1 (1965)

[26] A Zecca, Nuovo Cimento B 106, 413 (1991)

[27] J E Felten and R Isaacman, Rev Mod Phys 58, 689 (1986).

[28] D. Edwards, Monthly Notices of the Royal Astronomical Society, 159, 51 (1972).

[29] A. E. Romano and M. Sasaki, Gen. Rel. Grav. 44, 353 (2012) [arXiv:0905.3342 [astro-ph.CO]]. 
[30] M. N. Celerier, Astron. Astrophys. 353, 63 (2000) arXiv:astro-ph/9907206.

[31] C. Hellaby, PoS ISFTG, 005 (2009) [arXiv:0910.0350 [gr-qc]].

[32] V. Sahni and A. A. Starobinsky, Int. J. Mod. Phys. D 9, 373 (2000) astro-ph/9904398.

[33] V. Sahni, A. Shafieloo and A. A. Starobinsky, Phys. Rev. D 78, 103502 (2008) arXiv:0807.3548 [astro-ph]].

[34] C. Zunckel and C. Clarkson, Phys. Rev. Lett. 101, 181301 (2008) arXiv:0807.4304 [astro-ph]].

[35] A. A. Starobinsky, Phys. Lett. B 117, 175 (1982).

[36] A. A. Starobinsky, JETP Lett. 42, 152 (1985).

[37] M. Sasaki and E. D. Stewart, Progr. Theor. Phys. 95, 71 (1996) arXiv:astro-ph/9507001].

[38] N. Mustapha and C. Hellaby, Gen. Rel. Grav. 33, 455 (2001) astro-ph/0006083.

[39] D. H. Lyth, K. A. Malik and M. Sasaki, JCAP 0505, 004 (2005) astro-ph/0411220.

[40] R. A. Sussman, Class. Quant. Grav. 27, 175001 (2010) arXiv:1005.0717 [gr-qc]].

[41] A. Shafieloo, V. Sahni and A. A. Starobinsky, Phys. Rev. D 80, 101301 (R) (2009) arXiv:0903.5141 [astro-ph.CO]]. 

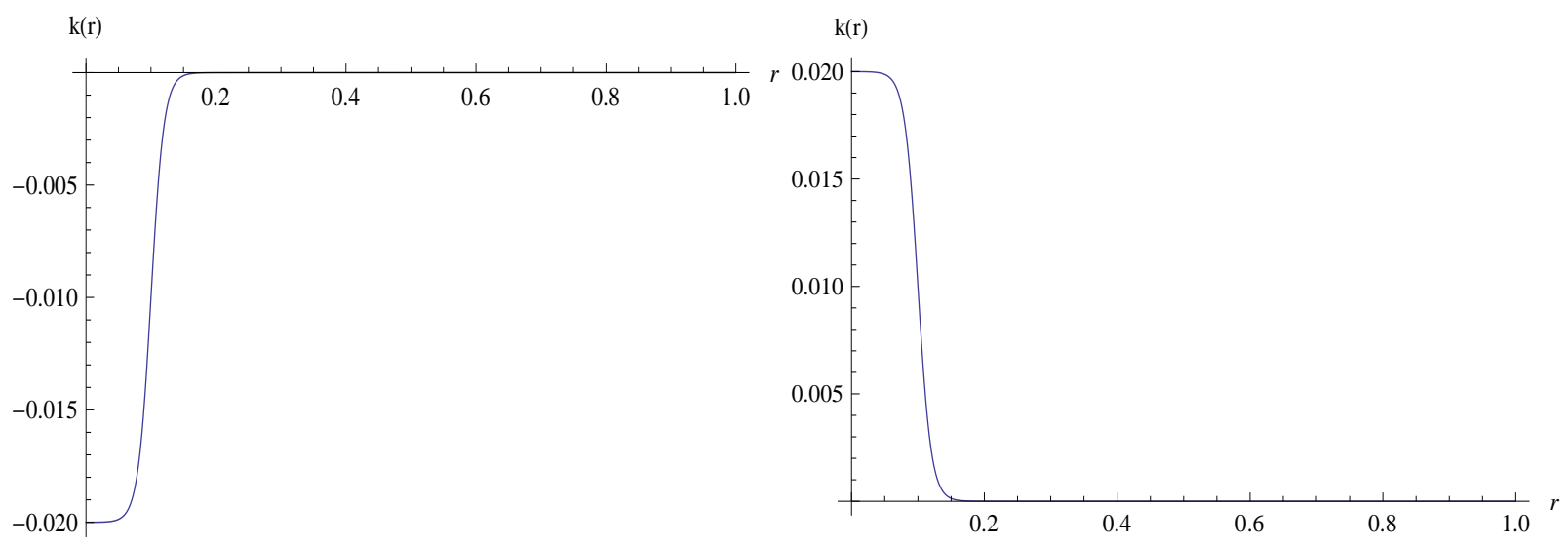

FIG. 1: $k(r)$ in units of $H_{0}^{2}$ is plotted for inhomogeneity of type $\mathrm{I}^{-}$and $\mathrm{I}^{+} . r$ is in units of $H_{0}^{-1}$.
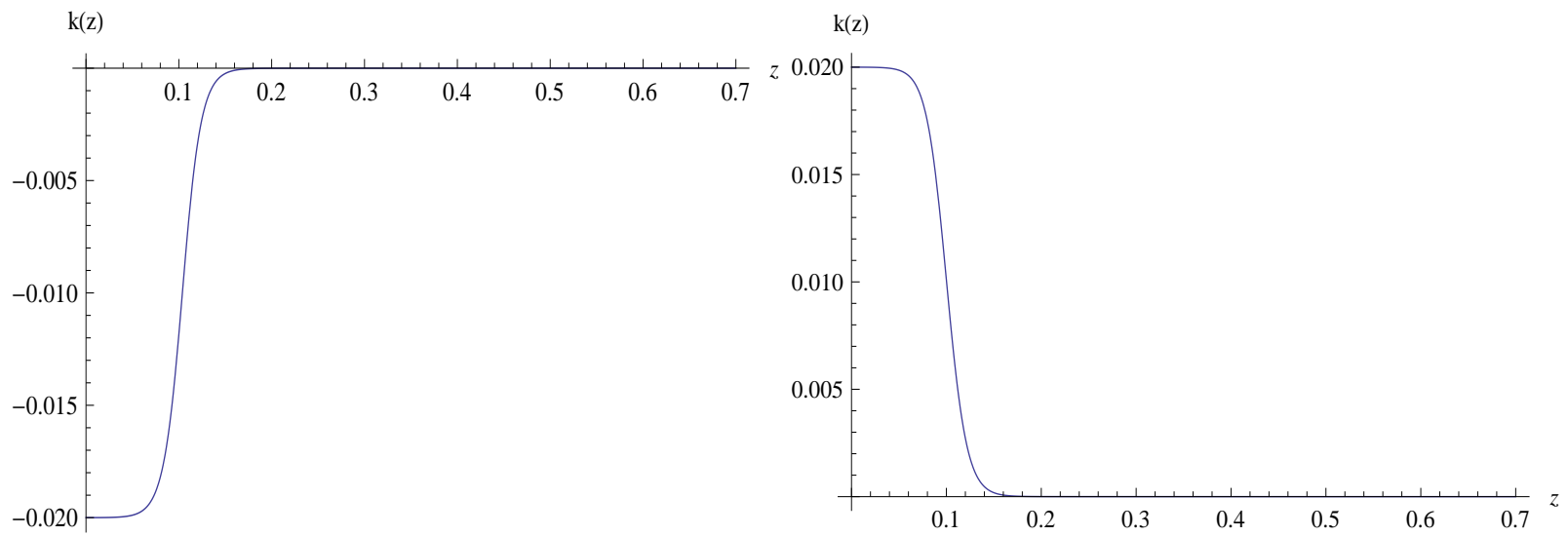

FIG. 2: $k(z)$ is plotted for inhomogeneity of type $\mathrm{I}^{-}$and $\mathrm{I}^{+}$.

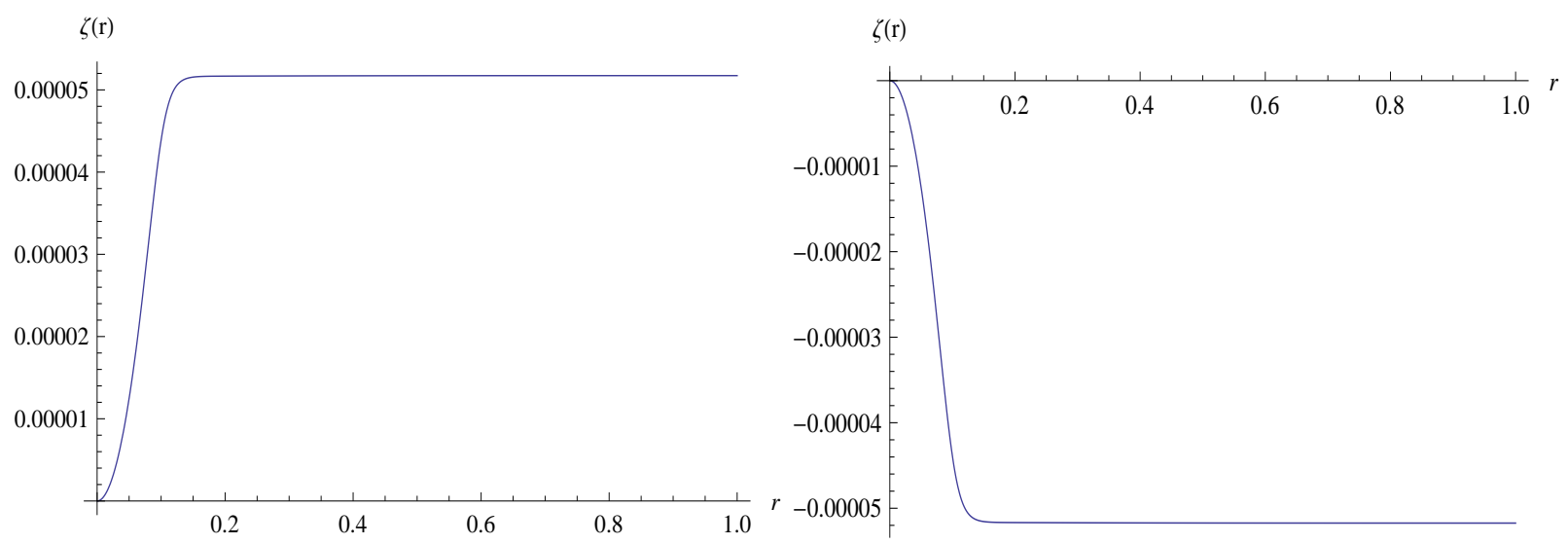

FIG. 3: $\zeta(r)$ is plotted for inhomogeneity of type $\mathrm{I}^{-}$and $\mathrm{I}^{+}$. 

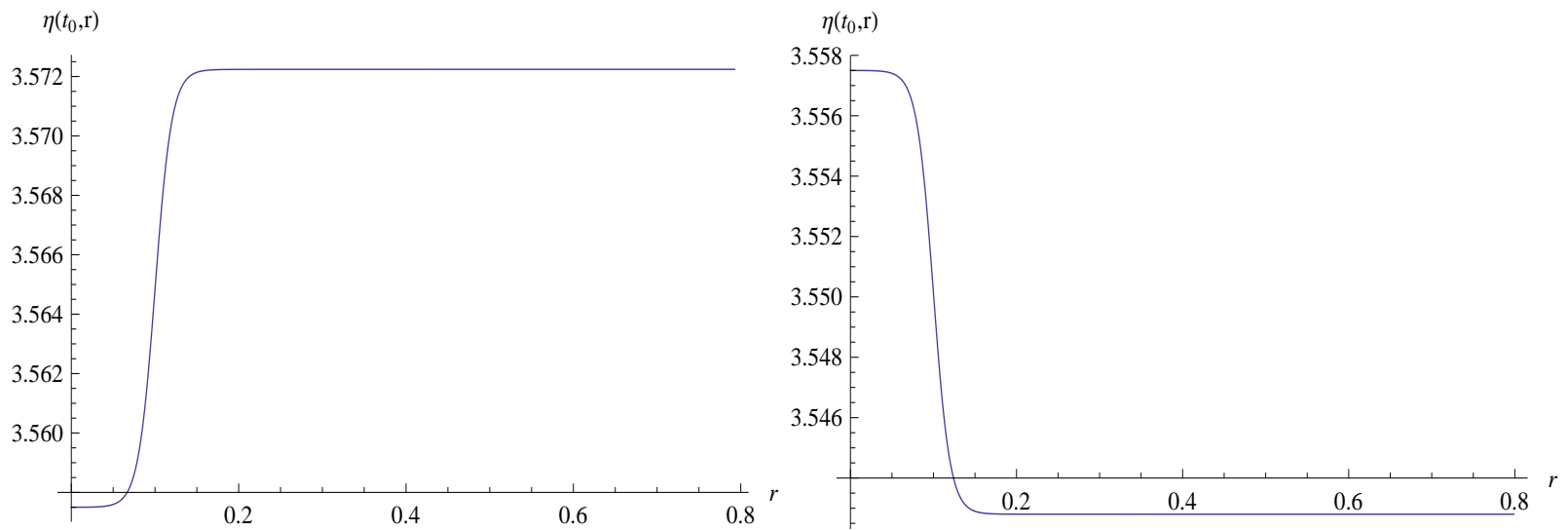

FIG. 4: $\quad \eta=v\left(t_{0}, r\right)$ in units of $H_{0}^{-1}$ is plotted for inhomogeneity of types $\mathrm{I}^{-}$and $\mathrm{I}^{+}$.

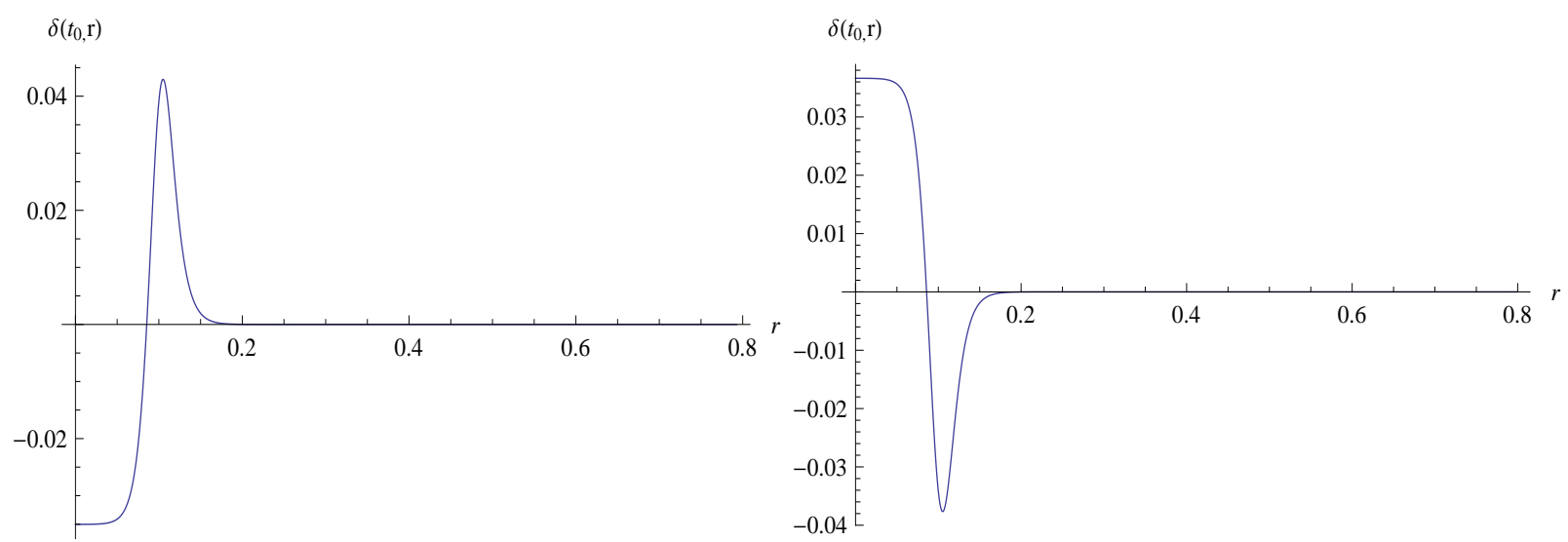

FIG. 5: $\delta\left(t_{0}, r\right)$ is plotted as a function of $r$ for inhomogeneity of types $\mathrm{I}^{-}$and $\mathrm{I}^{+}$.
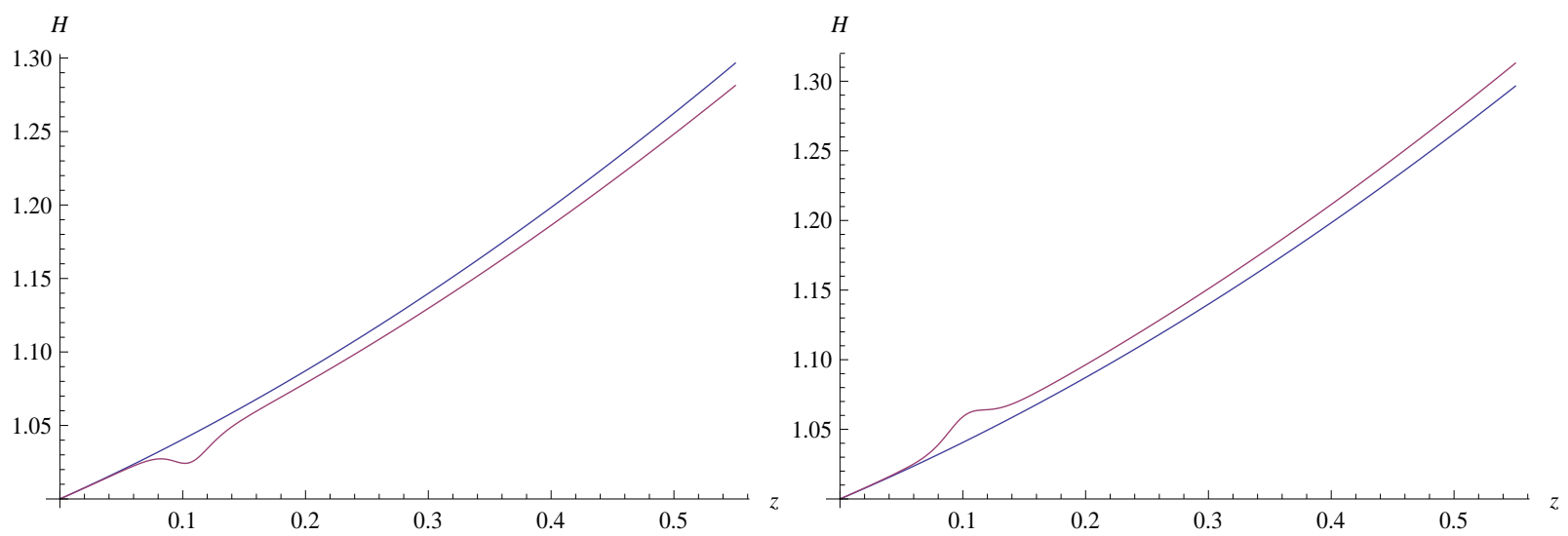

FIG. 6: $\quad H^{a p p}(z)$ is plotted for inhomogeneity of types $\mathrm{I}^{-}$and $\mathrm{I}^{+}$. 

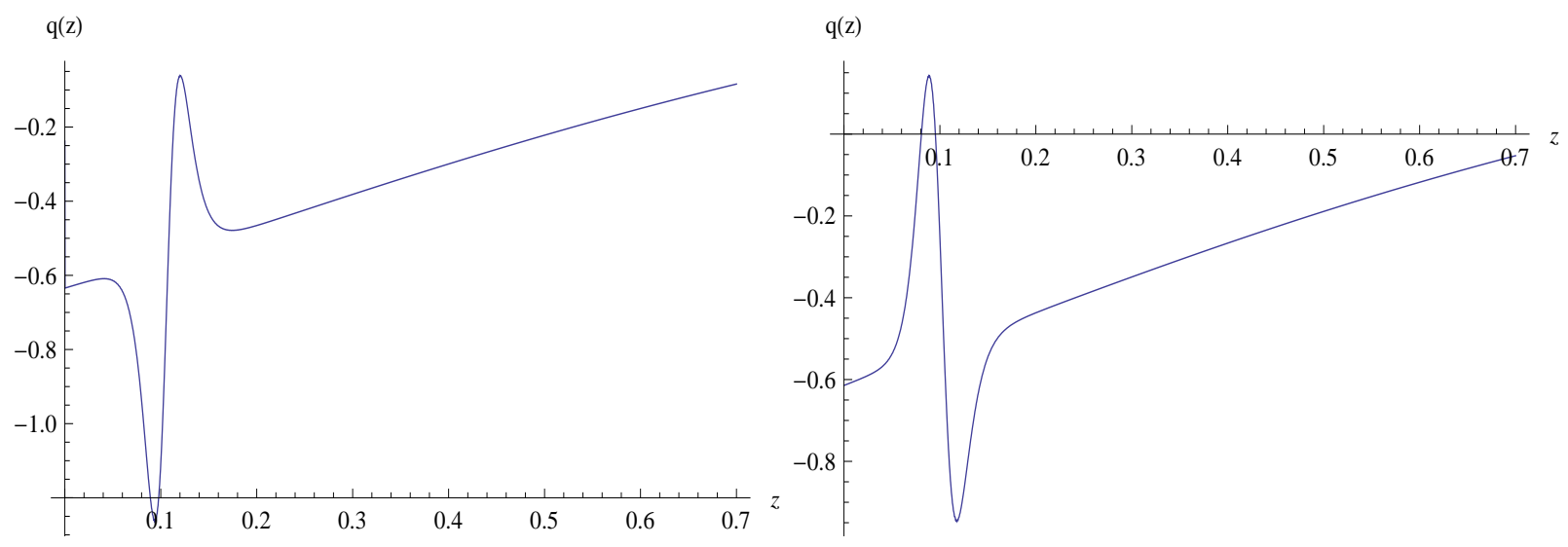

FIG. 7: $q^{a p p}(z)$ is plotted for inhomogeneity of types $\mathrm{I}^{-}$and $\mathrm{I}^{+}$.
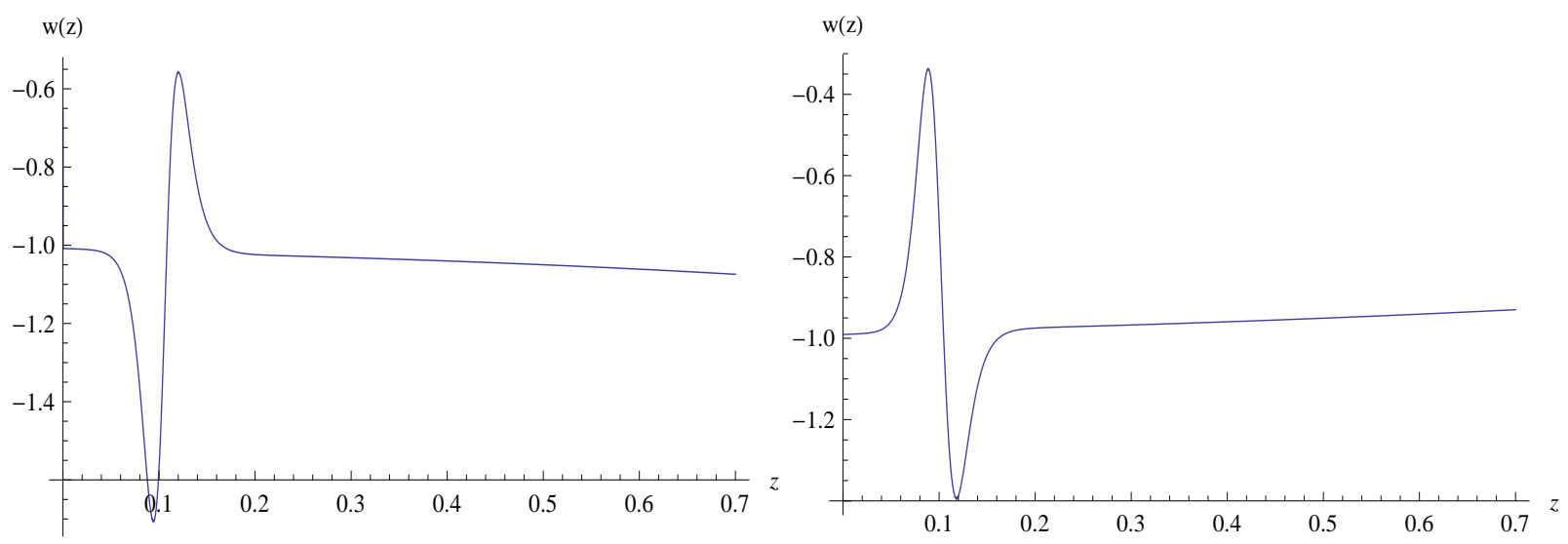

FIG. 8: $w_{D E}^{a p p}(z)$ is plotted for inhomogeneity of types $\mathrm{I}^{-}$and $\mathrm{I}^{+}$.
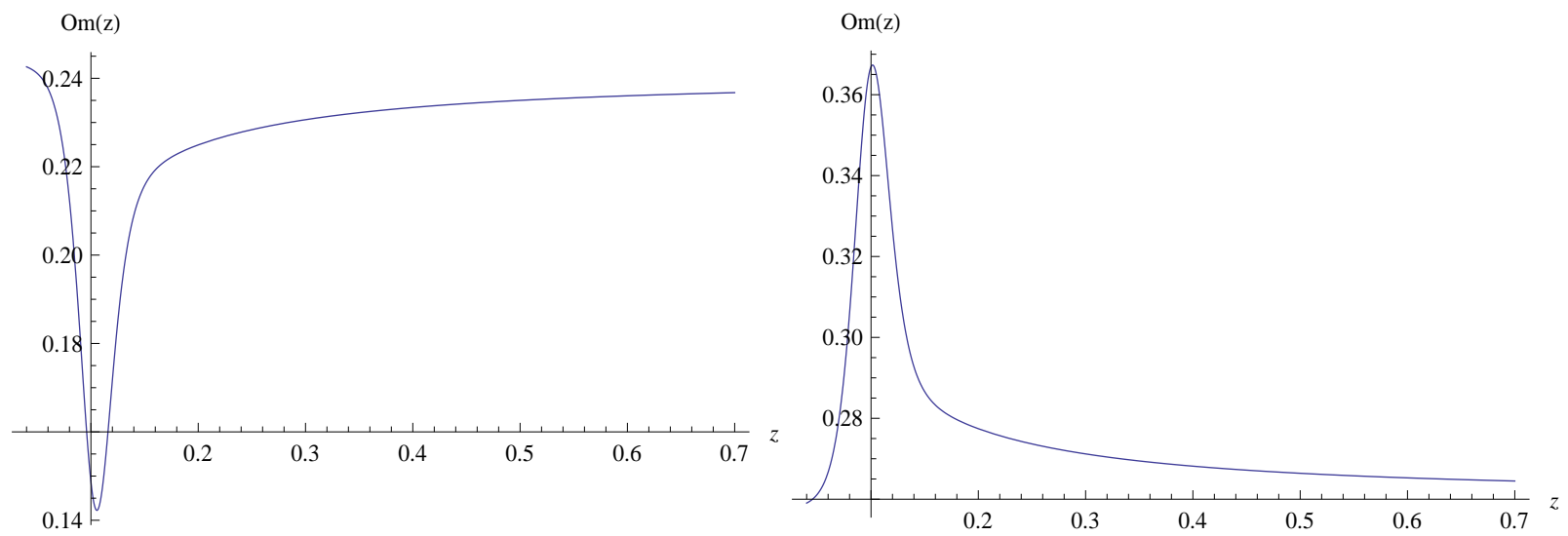

FIG. 9: $O m^{a p p}(z)$ is plotted for inhomogeneity of types $\mathrm{I}^{-}$and $\mathrm{I}^{+}$. 


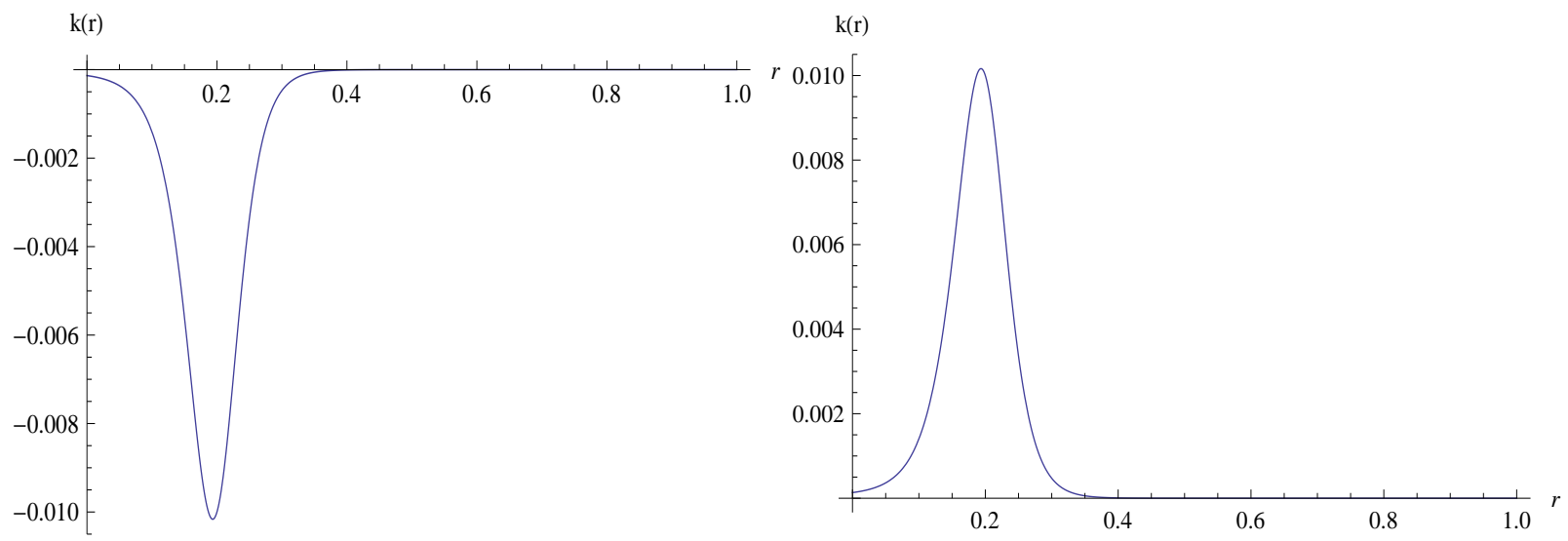

FIG. 10: The same as Fig. 1 but for inhomogeneity of types $\mathrm{II}^{-}$and $\mathrm{II}^{+}$.

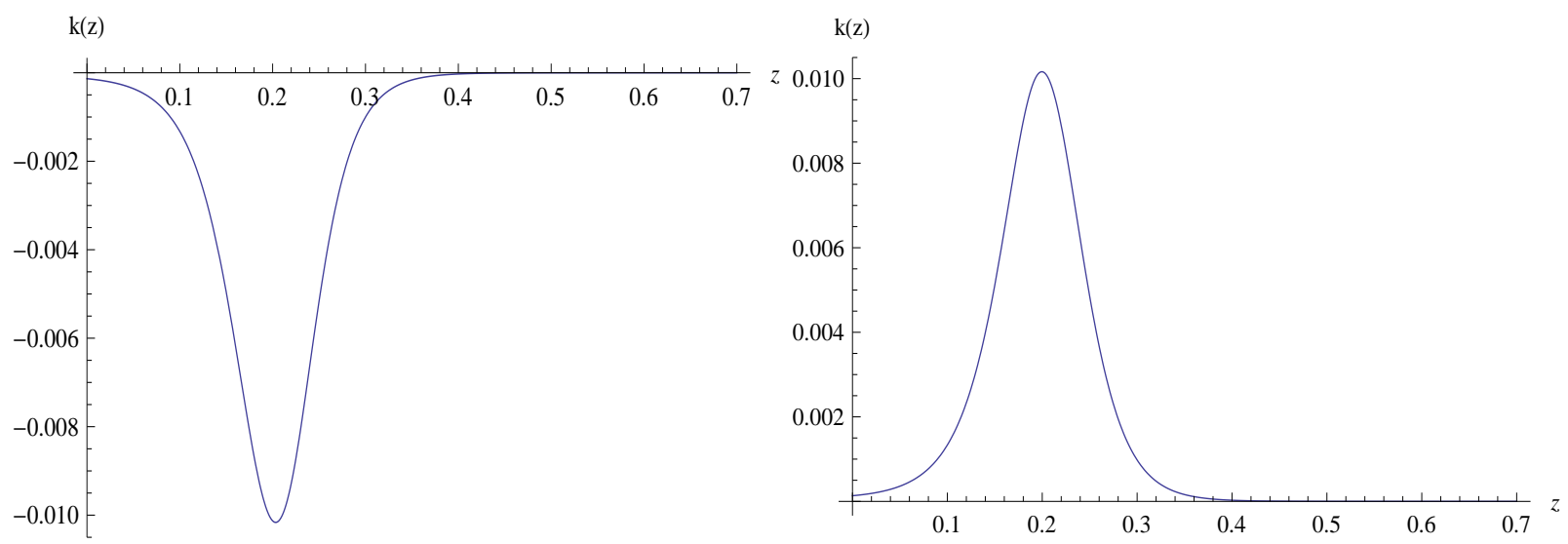

FIG. 11: The same as Fig. 2 but for inhomogeneity of types $\mathrm{II}^{-}$and $\mathrm{II}^{+}$.

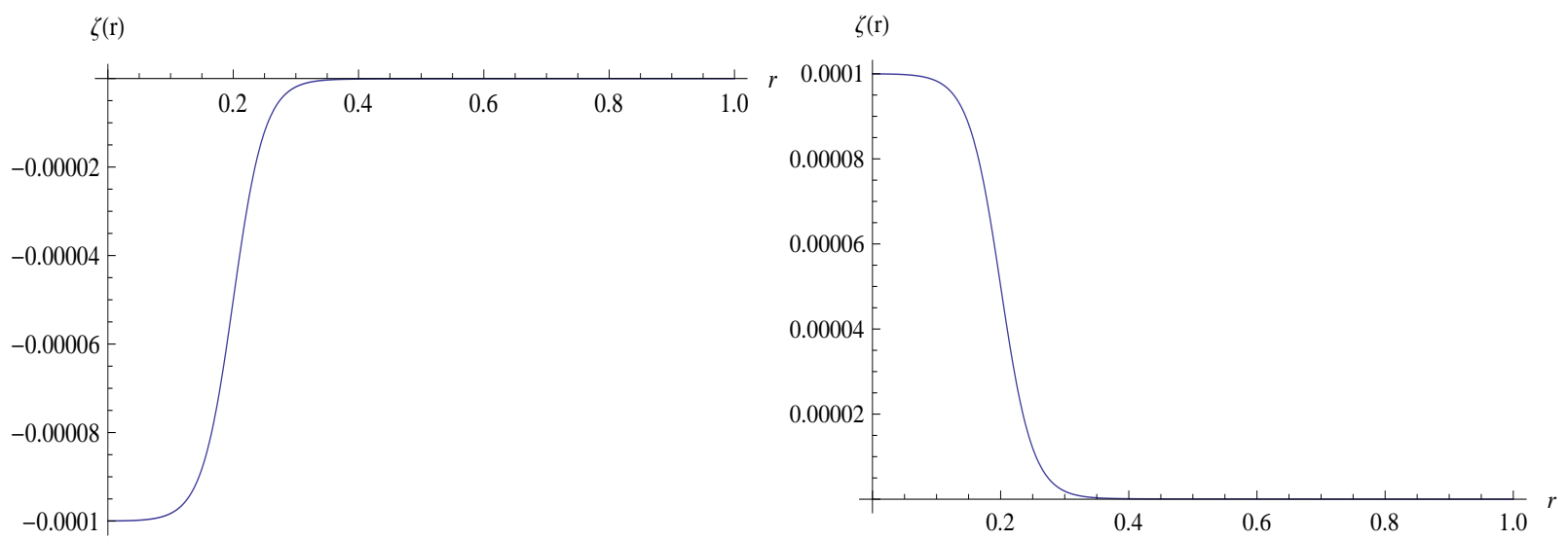

FIG. 12: The same as Fig. 3 but for inhomogeneity of types $\mathrm{II}^{-}$and $\mathrm{II}^{+}$. 

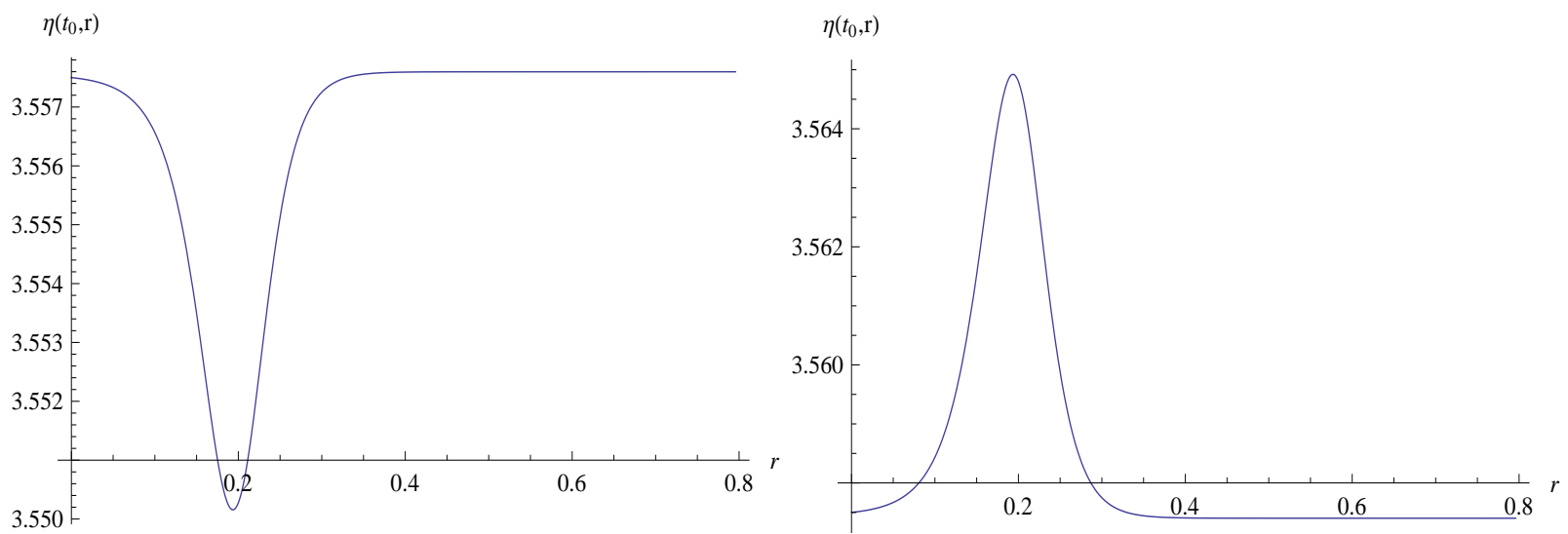

FIG. 13: The same as Fig. 4 but for inhomogeneity of types $\mathrm{II}^{-}$and $\mathrm{II}^{+}$.
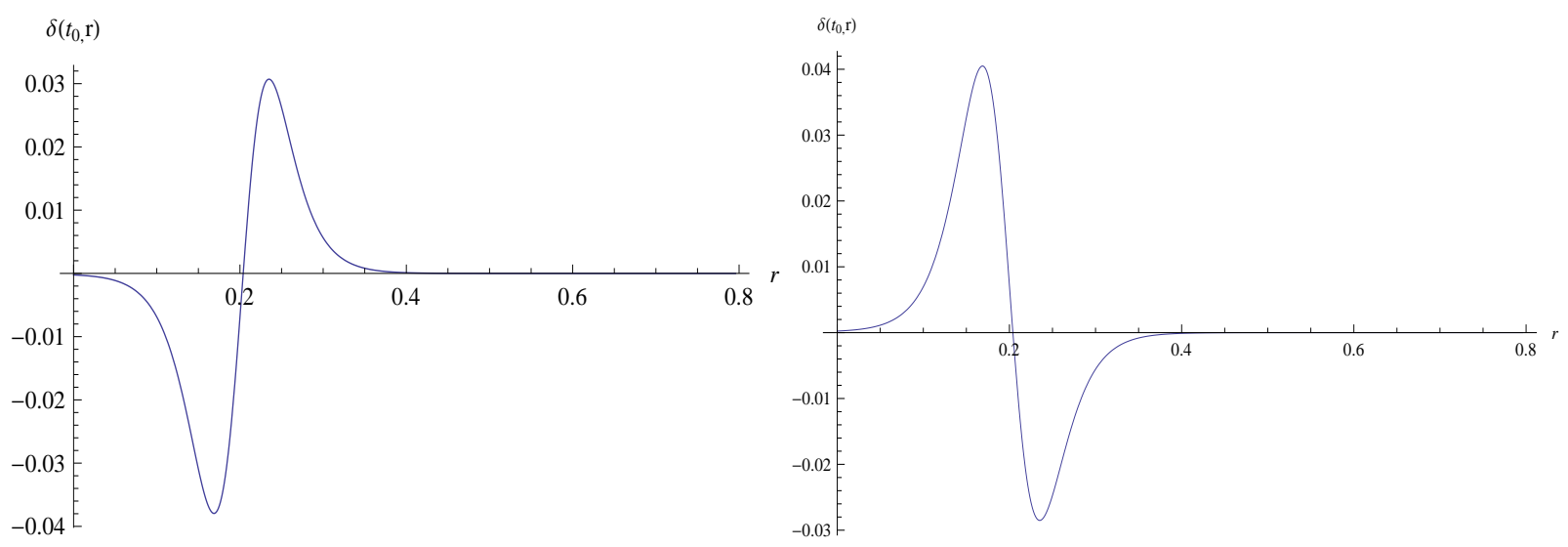

FIG. 14: The same as Fig. 5 but for inhomogeneity of types $\mathrm{II}^{-}$and $\mathrm{II}^{+}$.
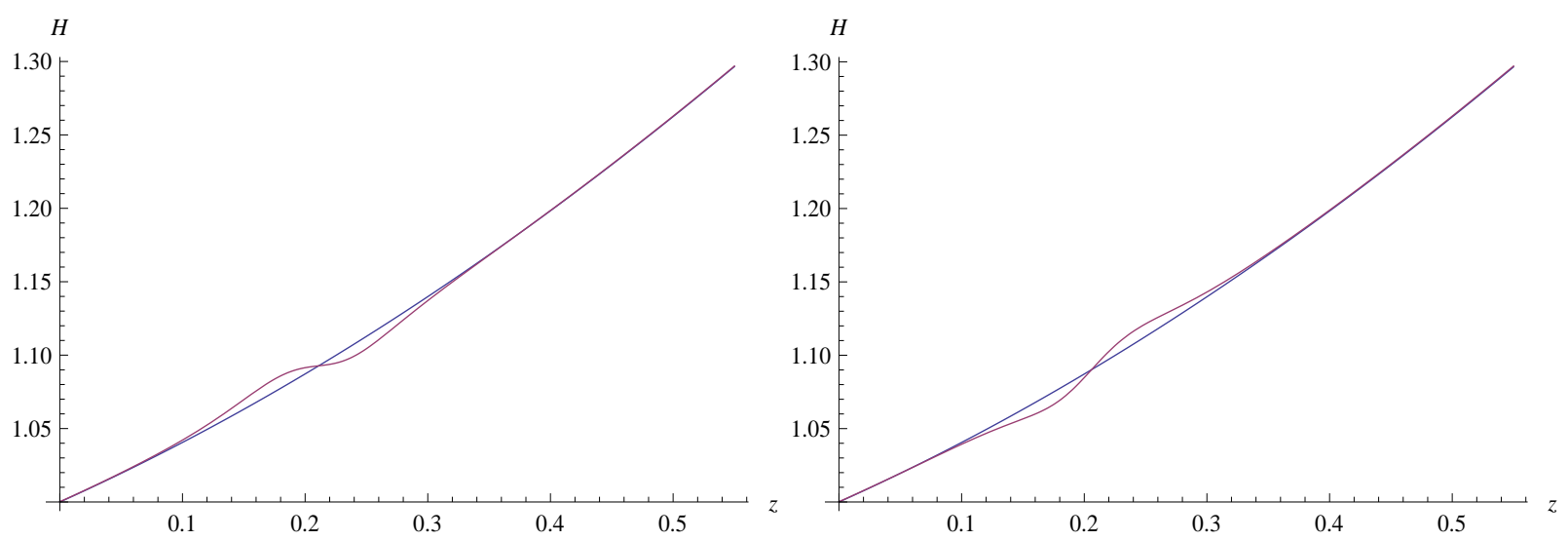

FIG. 15: The same as Fig. 6 but for inhomogeneity of types $\mathrm{II}^{-}$and $\mathrm{II}^{+}$. 

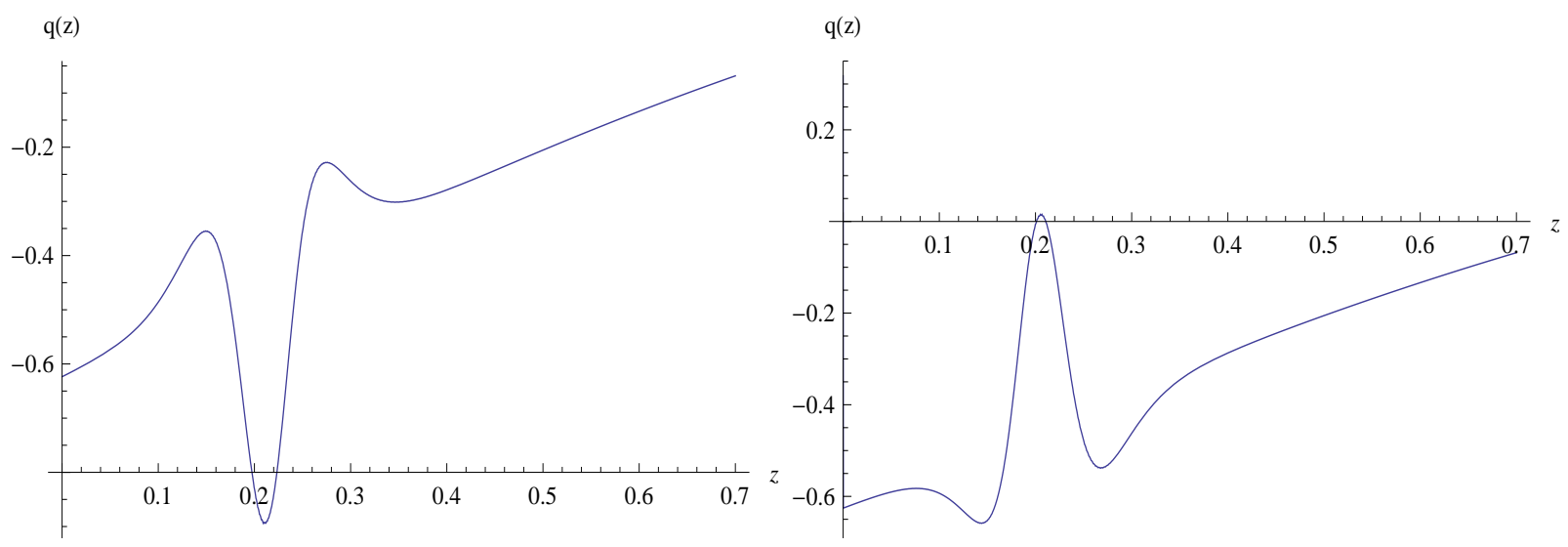

FIG. 16: The same as Fig. 7 but for inhomogeneity of types $\mathrm{II}^{-}$and $\mathrm{II}^{+}$.
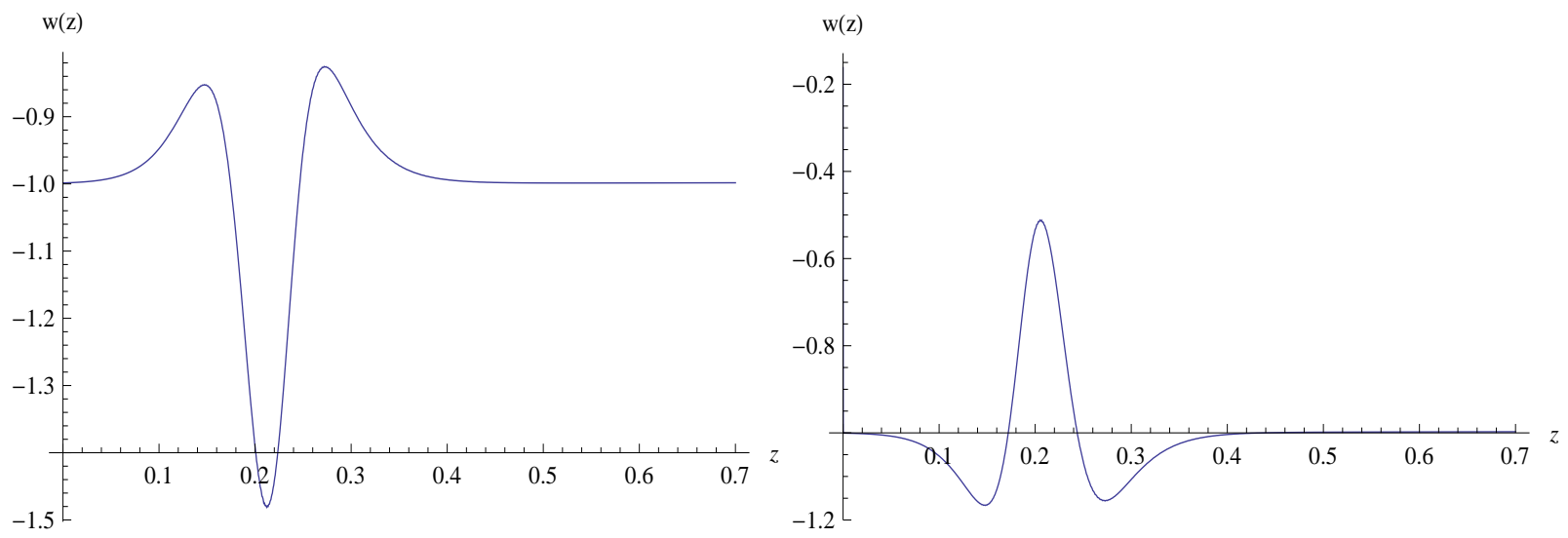

FIG. 17: The same as Fig. 8 but for inhomogeneity of types $\mathrm{II}^{-}$and $\mathrm{II}^{+}$.
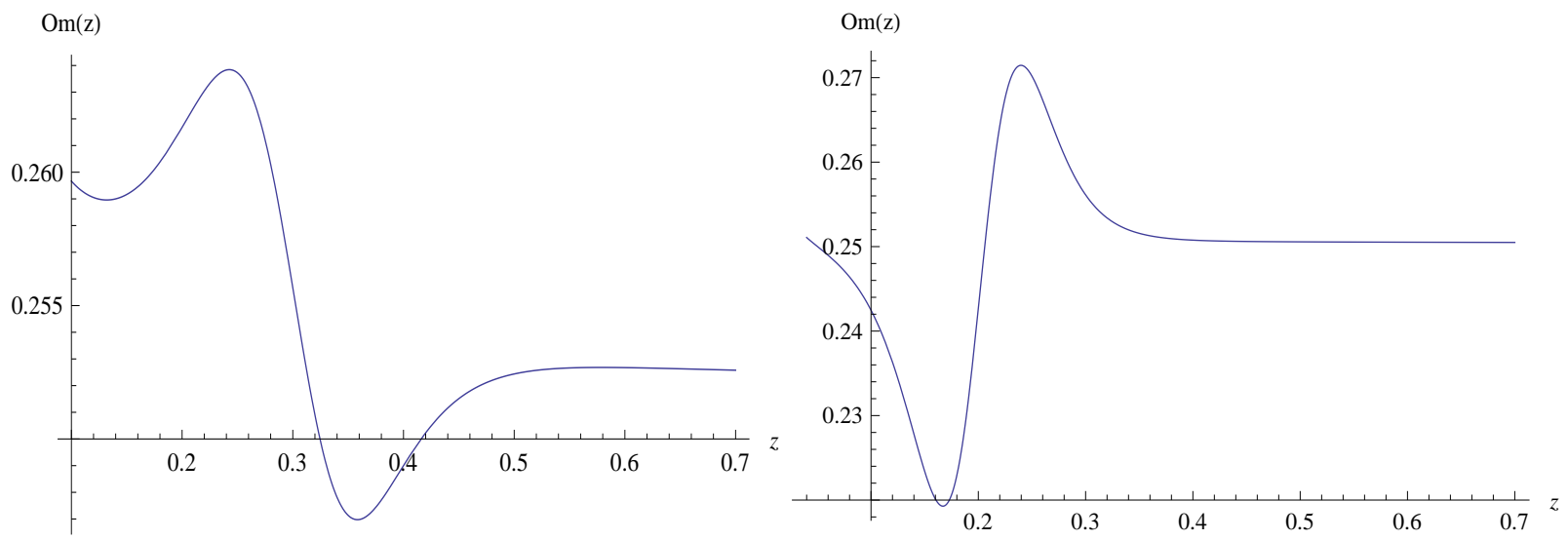

FIG. 18: The same as Fig. 9 but for inhomogeneity of types $\mathrm{II}^{-}$and $\mathrm{II}^{+}$. 


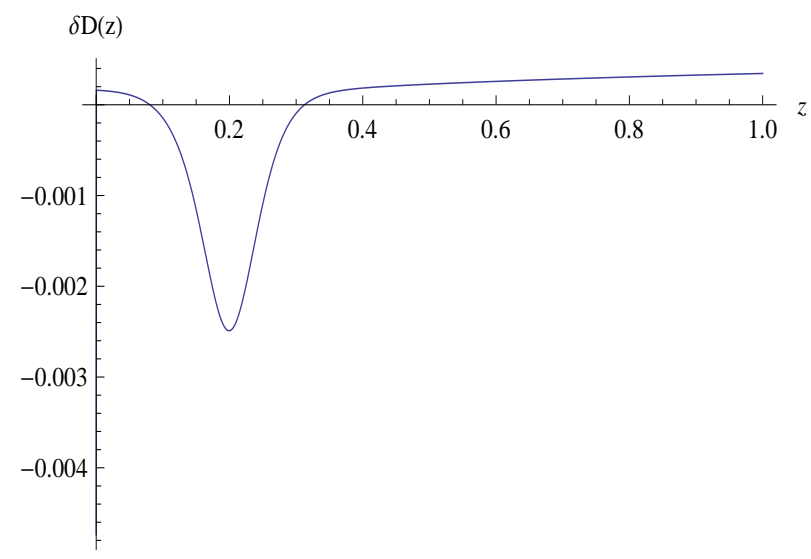

FIG. 19: $\delta D_{L}(z)=\left[D_{L}^{\Lambda C D M}(z)-D_{L}(z)\right] / D_{L}^{\Lambda C D M}(z)$, the relative difference between the luminosity distances, is plotted for model $\mathrm{II}^{+}$. As it can be seen the large scale inhomogeneities considered have a very small effect. 